\title{
Temperature Resistant Optimal Ratchet Transport
}

\author{
C. Manchein ${ }^{1}$, A. Celestino ${ }^{2}$ and M.W. Beims ${ }^{2,3}$ \\ ${ }^{1}$ Departamento de Física, Universidade do Estado de Santa Catarina, 89219-710 Joinville, Brazil \\ ${ }^{2}$ Departamento de Física, Universidade Federal do Paraná, 81531-980 Curitiba, Brazil and \\ ${ }^{3}$ Max-Planck-Institute for the Physics of Complex Systems, \\ Nöthnitzer Str. 38, 0118\%, Dresden, Germany, EU
}

(Dated: November 1, 2018)

\begin{abstract}
Stable periodic structures containing optimal ratchet transport, recently found in the parameter space dissipation versus ratchet parameter [PRL 106, 234101 (2011)], are shown to be resistant to reasonable temperatures, reinforcing the expectation that they are essential to explain the optimal ratchet transport in nature. Critical temperatures for their destruction, valid from the overdamping to close to the conservative limits, are obtained numerically and shown to be connected to the current efficiency, given here analytically. Results are demonstrated for a discrete ratchet model and generalized to the Langevin equation with an additional external oscillating force.
\end{abstract}

PACS numbers: 05.45.Ac,05.45.Pq

Keywords: Shrimps, ratchet currents, optimal transport, dissipation

Ratchets systems are remarkable profitable due their ability to displace particles without an external bias, which is known as ratchet transport. One of the challenges in the large variety of ratchet phenomena, and experiments, is to unveil how large currents of particles can be attained and controlled. If such displacement of particles can be controlled in distinct ratchet devices, it should be of great validity for technological applications. In this context we mention some experiments which successfully observed ratchet transport, namely cells to control cancer metastasis [1], solids and drops using the Leidenfrost effect [2,3], micro and nanofluids [4], particles in silicon membrane pores [5], cold atoms [6], among others. In addition, ratchet models appear as natural candidates to explain directed transport in biology [7], and have been realized in a wide range of physical systems [8]. In the real-world, ratchet systems always suffer environmental effects, like temperature, for example, which give rise to new current dynamics, and so need to be taken into account by any comprehensive approach. Therefore, to explain such transport in nature, and for technological applications, it is of fundamental priority to understand the effect of noise (thermal or not) on the efficiency of the ratchet current.

Recent works $[9,10]$ have shown that, in the absence of temperature, optimal ratchet currents $(\mathcal{R C} \mathrm{s})$ occur along organized structures, called isoperiodic stable structures (ISSs), in parameter spaces which involve the asymmetry, amplitude, phase of the ratchet and dissipation. The ISSs should play a fundamental role on the current generation in nature, since they bound the optimal $\mathcal{R C}$ region, delimit current reversal areas, and explain the remarkable diversity of possible $\mathcal{R C}$ s. Results have partially been extended to quantum systems [11]. The ISSs are Lyapunov stable islands and are supposed to be generic in dynamical systems [12-15]. Thus, any attempt to understand general effects of the temperature on the $\mathcal{R C}$, should be performed by analyzing the behavior of the
ISSs, and their surroundings, when the temperature is taken into account. This is the main goal of the present Letter: to show the transformations of the ISSs under temperature, and that optimal $\mathcal{R C}$ s inside the ISSs are resistant to reasonable temperatures. This is the opposite of what is done in non-inertial thermal rocket ratchets, where $\mathcal{R C}$ s are thermally activated. Our results also confirm that generic ISSs, like Shrimps discovered in deterministic dynamical systems [12, 13], for example, persist to some noise intensity in stochastic systems. Up to our knowledge, there is only one work [16] which observed the tendency that currents (not $\mathcal{R C} \mathrm{s}$ ) survive to reasonable noise effects inside ISSs.

A model which presents all essential features regarding unbiased $\mathcal{R} \mathcal{C}_{\mathrm{s}}$, is the dissipative map [6]

$$
\left\{\begin{array}{l}
p_{n+1}=\gamma p_{n}+K\left[\sin \left(x_{n}\right)+a \sin \left(2 x_{n}+\phi\right)\right]+\xi(n), \\
x_{n+1}=x_{n}+p_{n+1}
\end{array}\right.
$$

where $p_{n}$ is the momentum variable and $x_{n}$ de position, $n=1,2, \ldots, N$ represents the discrete time, $K$ is the nonlinearity parameter, and $\xi(n)$ is the stochastic variable obeying $\langle\xi(n)\rangle=0$ and $\left\langle\xi(n)^{2}\right\rangle=2(1-\gamma) k_{B} T$, with $k_{B}=1$ being the Boltzmann constant and $T$ the temperature. For $T=0$ this model was considered for $K=6.5, a=0.5,0 \leq \gamma<1$ in [17], and in the parameter spaces combining pairwise all parameters $\gamma, K, a, \phi$ $[9,10]$. The dissipation parameter $\gamma$ reaches the overdamping limit for $\gamma=0$ and the conservative limit for $\gamma=1$. The ratchet effect appears due to the spatial asymmetry, which occurs with $a \neq 0$ and $\phi \neq m \pi$ $(m=1,2, \ldots)$, in addition to the time reversal asymmetry for $\gamma \neq 1$. The above model is quite general to understand $\mathcal{R} \mathcal{C}_{\text {s. }}$. The parameter $K$ represents the intensity of an external asymmetric force in space. But since for $\gamma=1$ the above model can be derived from a kicked Hamiltonian, $K$ is as well the external kick which drives 
the system out of equilibrium, which is a necessary condition to obtain ratchet transport.

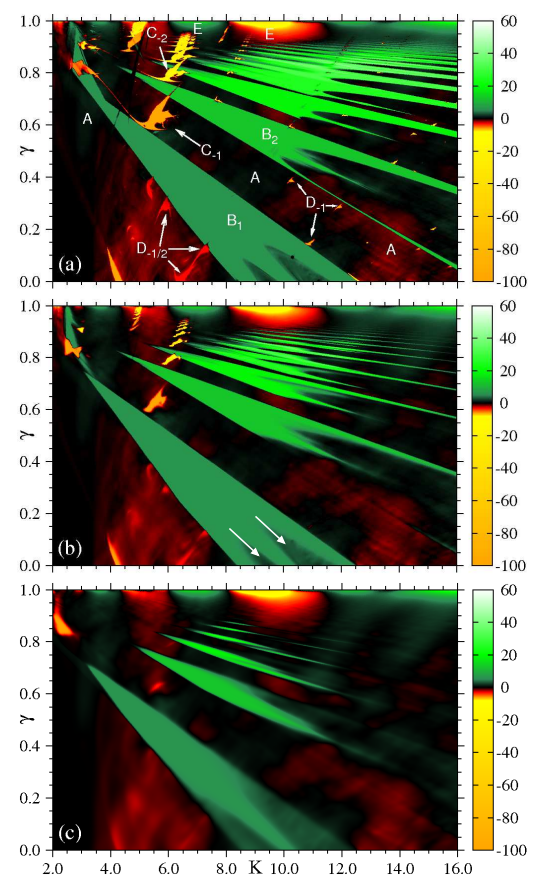

Figure 1. (Color online) The $\mathcal{R C}$ (see color bar) plotted in the parameter space $(K, \gamma)$ with a grid of $10^{3} \times 10^{3}$ points, $a=0.5, \phi=\pi / 2, M=10^{4}$ ICs with $\left\langle p_{0}\right\rangle=\left\langle x_{0}\right\rangle=0$ inside the unit cell $(-2 \pi, 2 \pi), N=10^{5}, n_{c}=9 \times 10^{4}$ iterations for (a) $T=10^{-5}$, (b) $T=10^{-3}$ and (c) $T=10^{-2}$

For a small temperature $T=10^{-5}$, Fig. 1(a) shows the $\mathcal{R C}=\frac{1}{M} \sum_{j=1}^{M}\left[\frac{1}{N-n_{c}} \sum_{n=n_{c}}^{N} p_{n}^{(j)}\right]$ (colors) as a function of $\gamma$ and $K . M$ is the number of initial conditions (ICs), $N$ is the total number of iterations used to calculate the $\mathcal{R C}$, and $n_{c}$ is obtained empirically and represents a cutoff value to avoid transient effects. The black color is related to small currents; dark green, green to white colors are related to increasing positive currents, while red to yellow colors related to increasing negative currents. Three main regions with distinct behaviors can be identified (for more details see Fig. 1 in [9] for $T=0$ ): $(i)$ a large "cloudy" background $A$, mixed with black, dark green and red colors, showing a mixture of zero, small positive and negative currents. This is a chaotic region [10] where the $\mathcal{R C}$ is not that large, neither efficient, and is independent of the magnitude of the positive Lyapunov exponent; ( $i i)$ structures $B_{L}, C_{L}$ and $D_{L}$ with sharp borders (the ISSs) and distinct colors, which are embedded in the cloudy background region ( $L$ is an integer or rational number). These ISSs are responsible for the larger $\mathcal{R C}$ s and they align themselves along preferred direction in the parameter space (see $[9,10])$. Inside the ISSs the Lyapunov exponents (LEs) are zero and negative, and the motion is periodic, while a sequence of doubling bifurcations cascade occurs in a specific direction. In the
$C_{L}$ and $D_{L}$ ISSs, these bifurcations start from the inner part [10] of the ISSs, and reach the chaotic motion at specific borders. In the $B_{L}$ ISSs, saddle-node bifurcations of period-1 occur on the left and right borders, and were determined analitically in $[9,10]$. Due to a crisis the analytical left borders do not match exactly with the numerical ones. A sequence of periodic doubling bifurcations occur when going down (smaller $\gamma$ ) to the right, where $K$ values increase [see white arrows in Fig. 1(b)]. It was also shown $[9,10]$ that the $\mathcal{R C}$ is independent on the period of the orbits. The values $2 \pi L$ determine the magnitude and direction of the $\mathcal{R} \mathcal{C}_{\mathrm{s}}$. For example, the $\mathcal{R C}$ inside the ISSs $B_{L}$, known as cuspidal singularities (cusps), increases positively along the sequence $B_{1}, B_{2}, \ldots$ The $\mathcal{R C}$ inside the $C_{L}$ ISSs, increases negatively along the sequence $C_{-1}, C_{-2}, \ldots$ The smaller $D_{L}$ ISSs are the shrimp-shaped, well known to appear in many dynamical systems. In general, it was shown for $T=0$ [10] that the $\mathcal{R C}$ is always more efficient inside the ISSs; (iii) the last region has strong positive and negative currents (region $E$ ), with not well defined borders, and occurs close to the conservative limit $\gamma=1$. This is related to a chaotic region (as shown in [6] for $T=0$ ), where the large $\mathcal{R} \mathcal{C}_{\mathrm{s}}$ are generated due to the accelerator modes which exist in the conservative limit. In Fig. 1(a) the temperature $T=10^{-5}$ is small and all ISSs are mainly preserved. However, the ISSs have small elongated antennae, as seen in Fig. 1 from [9], which already disappeared for this small $T$. Consequently, the lines connecting the distinct $D_{-1}$, and $D_{-1 / 2}$ ISSs, were destroyed by noise. LEs inside these antennae become positive, meaning that the ISSs antennae are destroyed.

Figure 1(b) shows the same parameter space, but now for a temperature $T=10^{-3}$. Comparing with Fig. 1(a), we observe that the lower thin antennae from the $B_{2}, B_{3}, \ldots$ ISSs were destroyed. The $C_{L}$ ISSs begin to be destroyed from their borders, which are not sharply defined anymore. The $D_{L}$ shrimp-shaped ISSs have almost disappeared, and the optimal $\mathcal{R} \mathcal{C}_{\mathrm{S}}$ inside them are substituted by the smaller $\mathcal{R} \mathcal{C}_{\text {s }}$ from the chaotic region. The magnitude of the $\mathcal{R C}$ s inside the remaining ISSs is essentially not affected by $T$, and still increases as the conservative limit is approached. All missing ISSs, or antennae, gave place to chaotic regions with smaller $\mathcal{R C}$ s. In the chaotic regions $A$ and $E$, the $\mathcal{R C}$ is almost unaffected by $T$.

As the temperature increases to $T=10^{-2}$, a significant amount of ISSs start to be destroyed. This is shown in Fig. 1(c). Almost all antennae from the $B_{L}$ ISSs are destroyed, remaining, in general, only the inner part of these structures, where the lowest period of the ISS is found. The less affected ISS is $B_{1}$, which originally had the smallest $\mathcal{R C}$ from the sequence $B_{1}, B_{2}, \ldots$ The boundaries of these ISSs are not sharp anymore, but appear blurred. Remarkable is that the magnitude of the $\mathcal{R C} \mathrm{s}$, inside the inner part of the remaining $B_{L}$ ISSs, is 
almost the same. Along the period doubling bifurcations [see white arrows in Fig. 1(b)], $T$ destroys the optimal $\mathcal{R C}$, remaining just a region (now chaotic) with $\mathcal{R C} \mathrm{s}$ values a tiny amount larger when compared to the surrounding background chaotic region. Besides that, the $C_{L}$ ISSs are completely destroyed, giving place to the chaotic motion with almost zero current. Comparing to Fig. 1(a), in the chaotic regions $A$ and $E$ the $\mathcal{R C}$ s remain unchanged. Close to $K \sim 2.5$ and $\gamma \sim 0.9$, we observe in Fig. 1(c) a region where larger $\mathcal{R C}$ s occur. These larger $\mathcal{R C}$ s were thermally activated, since they do not show up in Figs. 1(a)-(b). It gives an example, in distinction to all other regions of the explored parameter space, where the $\mathcal{R C}$ is activated by $T$, instead of being destroyed.

In this context the key question is: what is the critical $T$ to destroy the $\mathcal{R C}$ in different stable regions (ISSs) of the parameter space? To answer this question the following simulation was performed. For each parameter combination $(K, \gamma), T$ was increased until the $\mathcal{R C}$ was close to zero, within the precision $\Delta \sim \pm 3.0$, which is a value bellow the minimum $\mathcal{R C}$ allowed by the periodic constraints in ISSs with main periods 1 and 2 [9], that is, the main part of our parameter space. It is possible to observe that the close to zero $\mathcal{R C}$ in the chaotic region, is independent of the parameters $(K, \gamma)$ within the precision $\Delta$. This defines the critical temperature $T_{c}$, where the optimal $\mathcal{R C}$, due to periodic motion (ISSs), is transformed into the small $\mathcal{R C}$ due to the chaotic motion. Figure 2(a) shows $T_{c}$ (color bar) in the parameter space

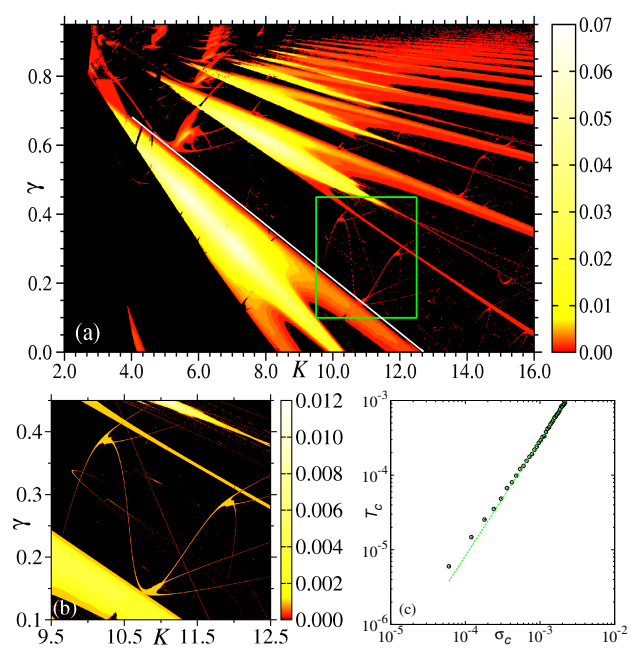

Figure 2. (Color online) The critical temperature $T_{c}$ (see color bar) necessary to destroy the optimal $\mathcal{R C}$ plotted in the parameter space (a) from Fig. 1 and (b) inside the box from Fig. 2(a) and for $a=0.5, \phi=\pi / 2$ and same ICs from Fig. 1. In (c) we show that $T_{c} \sim\left|\gamma-\gamma_{c}\right|^{1.5}$, for $K=2 \pi$ and around $\gamma_{c}=0.5$.

$(K, \gamma) . T_{c}$ increases from black $\left(T_{c} \sim 0.0\right)$ to red, yellow and white $\left(T_{c} \sim 0.07\right)$. It is clearly observed that $T_{c}$ is larger inside the ISSs, especially inside their inner parts.
Figure 2(b) shows in details $T_{c}$ from the box of Fig. 2(a), with three connected shrimp-like structures. Thus, the ISSs can still be recognized in such $T_{c}$ analysis. Even though $T_{c}$ is larger inside the inner parts of the ISSs, it tends to decrease at their borders.

The physical origin of the thermally induced destruction of the optimal $\mathcal{R C}$ can be rather complex. It depends very much on which region in the parameter space is considered. Inside the ISSs there is always at least one periodic stable attractor, which coexists with the unstable periodic orbits and chaotic repellers. With the introduction of $T$, the attracting motion (periodic or not) becomes transient and the dynamics is ergodic, as earlier studies about noise effects and transient chaos in dynamical systems indicate [18]. Temperature also induces transitions between all possible dynamics: periodic/non-periodic attractors, chaotic repellers etc. Thus, for $T \neq 0$, the final $\mathcal{R C}$ s will be an average over the velocities of each of such dynamics, weighted by their lifetimes. Since the $\mathcal{R C}$ is more efficient inside the ISSs [10], we should understand the effect of $T$ on the $\mathcal{R C}$ s efficiency, which is defined by $\eta=\langle\langle p\rangle\rangle^{2} /\left|\left\langle\left\langle p^{2}\right\rangle\right\rangle-D_{0}\right|[19] . D_{0}=k_{B} T$ is a measure of the thermal energy and $\langle\langle\ldots\rangle\rangle$ denotes an average over ICs, thermal realizations and time. For the ergodic case it reduces to just one average, over the asymptotic distribution. Considering that the thermal "kicks" are typically weak enough, so they can be neglected after one iteration, but rarely strong enough to drive the orbit out of the attractor, then it is possible to show that

$$
\eta=\frac{\left(\sum_{i} \alpha_{i} \mathcal{R C}^{(i)}\right)^{2}}{\left|\sum_{i} \alpha_{i}\left\langle p_{i, T=0}^{2}\right\rangle+(1-\gamma) D_{0} /(1+\gamma)\right|},
$$

where the sum is over all attractors, $\left\langle p_{i, T=0}^{2}\right\rangle$ is the mean of the momentum square on the attractor $i$ (for $T=0), \alpha_{i}=\mu_{i} \tau_{i}$ is the statistical weight of each attractor, where $\tau_{i}$ is the mean lifetime on attractor $i$, and $\mu_{i}=\lim _{n \rightarrow \infty}\left\langle m_{i}\right\rangle / n$, where $m_{i}$ is the number of times a given trajectory visited the attractor $i$ during the time $n$. According to our simulations, transitions between deterministic dynamics [the sums over attractors from Eq. (2)], induced by $T$, affect more the efficiency than pure thermal effects (proportional to $D_{0}$ ). Such transitions may even increase the efficiency by allowing the orbit to access attractors with higher currents related to the unstable dynamics from $T=0$, as could be seen in Fig. 1(c). However, inside the ISSs the current is usually optimal, thus $T$ tends to decrease its efficiency. Also, as $T_{c}$ is related to very frequent transitions between different dynamics, the efficiency for larger $T$ must decrease substantially.

As far as we have knowledge, there is no first principles theory that quantifies the critical temperatures which destroy the $\mathcal{R C}$ s. Quasipotentials [18] and escape rates 
[16] from periodic/non-periodic attractors and chaotic repellers, have been used to quantify $T_{c}$ in a context not related to $\mathcal{R C}$ s. It is known [18] that close to a saddle-node bifurcation, the dynamics of a higher-dimensional system can be reduced to an one-dimensional normal form, and then the critical Gaussian noise follows $\sigma_{c} \sim\left|\gamma-\gamma_{c}\right|^{3 / 4}$, with $\sigma_{c}$ being the critical noise amplitude. To check this, we plot $T_{c}$ in Fig. 2(c) as a function of $\left|\gamma-\gamma_{c}\right|$, for $K=2 \pi$ and $\gamma_{c}=0.5$. This is a point at the border line from the $B_{1}$ ISS (see white line in Fig. 2(a)), which was calculated analytically in $[9,10]$, and has a saddle-node bifurcation. Since in our case $\sigma \propto \sqrt{T}$ we expect that $T_{c} \sim\left|\gamma-\gamma_{c}\right|^{3 / 2}$. This is exactly what is observed in Fig. 2(c) from our numerical simulation (cross points). For comparison, we plotted the exact continuous line $\left|\gamma-\gamma_{c}\right|^{3 / 2}$.

To generalize our results to a continuous, and more realistic system, we analyze the $T_{c}$ to destroy the optimal $\mathcal{R C}$ in the Langevin equation: $\ddot{x}+\gamma \dot{x}-$ $5.0[\sin (x)+0.7 \cos (2 x)]-K_{t} \sin (t)+\xi(t)=0$, where $K_{t}$ is the amplitude of the external time oscillating force, $\gamma$ is the viscosity, while the ratchet potential and the stochastic term $\xi(t)$ are like from Eq. (1). ISSs have been found [9] for this problem for $T=0$ in the parameter space $\left[K_{t}, \chi=e^{(-\gamma)}\right]$. Figure 3 shows the critical temperature

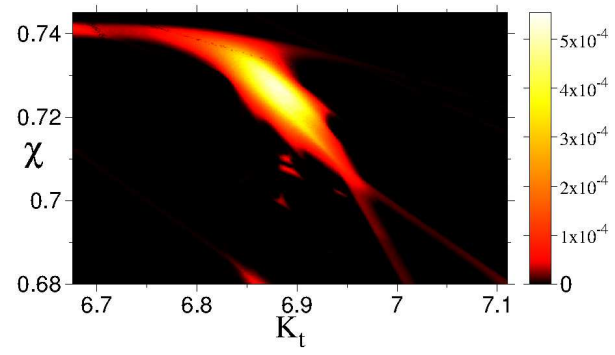

Figure 3. (Color online) The critical temperature $T_{c}$ in the parameter space $\left(K_{t}, \chi=e^{(-\gamma)}\right)$ of the Langevin equation. The shrimp-like ISS can be identified.

$T_{c}$, required to destroy the $\mathcal{R C}$ in a portion of the parameter space $\left(K_{t}, \chi\right)$. The black color $\left(T_{c}=0\right)$ means that no optimal $\mathcal{R C}$ is observed, red, yellow to white colors are related to the increasing values of $T_{c}$ required to destroy the $\mathcal{R C}$. The shrimp-like ISS can be identified, showing that its inner part is more resistant (yellow) to external noise, while on its border (red), smaller noises are enough to destroy the optimal $\mathcal{R} \mathcal{C}_{\mathrm{s}}$ region.

Concluding, optimal ISSs $\mathcal{R} \mathcal{C}_{\mathrm{s}}$ in the parameter space are shown to be resistant to reasonable temperatures $T$, i.e., it exists for thermal energies around 1000 times smaller then the transport energy. Increasing the values of $T$, the $\mathcal{R} \mathcal{C}_{\mathrm{s}}$ inside the ISSs begin to be destroyed from their borders, and the ISSs start to become more and more blurred. We determined numerically the critical temperature $T_{c}$, required to destroy the optimal $\mathcal{R C} \mathrm{s}$ in the whole parameter space. Results show that, in general, as nearer of the ISSs' center, higher are the critical temperatures. The ISSs persist in such a plot. Distinct regions in the parameter space present different dynamics and $T$ affects the optimal $\mathcal{R C}$ s distinguishable. The general physical explanation is that $T$ induces a transient chaos and also transitions between all possible dynamics: periodic and non-periodic attractors, chaotic repellers etc. An analytical expression for the $\mathcal{R C}$ efficiency was obtained, which combines all these dynamics. It essentially shows that there are two thermal effects on the efficiency, namely the slight variation in $\left\langle p^{2}\right\rangle$ inside the attractor, and the transitions between different dynamics. Considering the scale of $T_{c}$, the first effect is practically neglectable and thus the $\mathcal{R C}$ is optimal inside the ISSs, where the transitions are very rare. For $T \neq 0$, the periodic attractor loses its dominance on the $\mathcal{R C}$, allowing the rise of those new kinds of dynamics that control the $\mathcal{R C}$ above a critical value of $T, T_{c}$. This temperature is obtained numerically in Fig. 2 for the asymmetric standard map, in Fig. 3 for the Langevin equation with an external driving force, and is a direct measure of all complicated combined effects to destroy the $\mathcal{R C}$. It also gives quantitative information about the "global stability" of attractors under noise in phase space. This allows us to relate the robustness of the optimal $\mathrm{RC}$ to the global stability of solutions, rather than to the local stability obtained from the Lyapunov analysis. As well motivated by [20], information regarding global stability is usually more relevant in real-world systems where perturbations are finite. Hence, our results underscore the fundamental role of isoperiodic stable structures in the real-world optimal ratchet transport, where environmental effects cannot be neglected.

[1] G.Mahmud et al., Nature Physics 5, 606 (2009).

[2] G.Lagubeau et al. , Nature Physics 7, 395 (2011).

[3] H. Linke et al., Phys. Rev. Lett. 96, 154502 (2006).

[4] T.Kulrattanarak et al., Microfluidics and Nanofluidics 10, 843 (2011).

[5] S.Matthias and F.Müller, Nature 424, 53 (2003).

[6] G.G.Carlo et al., Phys.Rev.Lett. 94, 164101 (2005).

[7] F.Jülicher, A.Ajdari, and J.Prost, Rev.Mod.Phys. 1997, 1269 (1997).

[8] P.Reimann, Phys.Rep. 361, 57 (2002).

[9] A.Celestino et al., Phys.Rev.Lett. 106, 234101 (2011).

[10] A.Celestino et al., (arXiv:1111.1420).

[11] G.G.Carlo, Phys.Rev.Lett. 108, 210605 (2012).

[12] J.A.C.Gallas, Phys.Rev.Lett. 70, 2714 (1993).

[13] S.Hayes et al., Phys.Rev.Lett. 70, 3031 (1993).

[14] C.Bonatto and J.A.C.Gallas, Phys.Rev.E 75, R055204 (2007).

[15] R.Stoop et al. , Phys.Rev.Lett. 105, 074102 (2010).

[16] D.Speer, R.Eichhorn, and P.Reimann, Phys.Rev.E 76, 051110 (2007).

[17] L. Wang et al., Phys.Rev.Lett. 99, 244101 (2007).

[18] Y-C.Lai and T.Tél, Transient Chaos: Complex Dynamics in Finite Time Scales, vol. 173 (Springer, 2011).

[19] L.Machura et al., J.Phys.:Cond.Matter 17, S3741 (2005). 
[20] P. Beale, Phys.Rev.A 40, 3998 (1989). 